\title{
Genre et mixité : défis pour l'éducation physique au Brésil
}

\section{Helena Altmann}

\section{(2) OpenEdition \\ 1 Journals}

\section{Édition électronique}

URL : http://journals.openedition.org/trema/1145

DOI : 10.4000/trema. 1145

ISSN : 2107-0997

\section{Éditeur}

Faculté d'Éducation de l'université de Montpellier

\section{Édition imprimée}

Date de publication : 1 juin 2010

Pagination : 111-122

ISSN : 1167-315X

\section{Référence électronique}

Helena Altmann, «Genre et mixité : défis pour l'éducation physique au Brésil », Tréma [En ligne],

32 | 2010, mis en ligne le 01 juin 2012, consulté le 04 mai 2019. URL : http://journals.openedition.org/ trema/1145; DOl : 10.4000/trema.1145

Ce document a été généré automatiquement le 4 mai 2019.

Trema 


\title{
Genre et mixité : défis pour l'éducation physique au Brésil
}

\author{
Helena Altmann
}

\section{Introduction}

1 Tout au long de l'histoire, l'école a mené un grand nombre d'actions distinctives de classification et de hiérarchisation des sujets, à l'exemple des pratiques de distinction garçons / filles et hommes / femmes. De même, les cours et les programmes scolaires produisent des différences de genre, séparent ou mélangent, classifient, hiérarchisent, offrent des expériences et des apprentissages distincts aux hommes et aux femmes. Au sein de l'école, l'éducation physique est l'un des espaces où ces distinctions de genre sont les plus visibles. Envisagée au Brésil comme une discipline pratique qui travaille avec le corps, l'éducation physique justifie les ségrégations à travers les différences biologiques entre hommes et femmes. Ces ségrégations sont renforcées par des pratiques de signification autour du sport, devenu depuis la décennie de 1940 le principal contenu de cette discipline.

2 Toutefois, les années 1990 ont connu dans le pays des changements importants en termes de genre, d'éducation et d'éducation physique, aussi bien au niveau de la recherche que des pratiques scolaires. En plus de l'augmentation d'études sur le genre, des modifications des législations et des pratiques ont mis fin à la ségrégation des filles et des garçons dans les classes d'éducation physique, permettant une interaction entre les genres qui n'est pas sans provoquer des conflits et des résistances.

3 Le présent article analyse certains des défis scolaires qui ont accompagné cette configuration mixte de l'éducation physique. Le changement dans l'organisation des cours d'éducation physique a coïncidé au Brésil avec l'apparition des études sur le genre. Il ne s'agit cependant pas d'une relation de cause à effet, mais le résultat de raisons économiques pour pouvoir réduire les coûts des enseignants. Si dans le passé une seule classe avait besoin de deux enseignants - en général un pour les filles et un autre pour les 
garçons - désormais un seul devenait suffisant. La catégorie Genre fut un outil analytique important dans les débats sur cette question. Partant de là, nous tenterons d'analyser quelques-uns des défis de genre auxquels sont confrontées les classes d'éducation physique mixtes ${ }^{1}$ travaillant avec des contenus sportifs, et ce à partir de deux axes d'analyse : l'occupation de l'espace physique scolaire et les exclusions dans les sports.

\section{Classes mixtes et genre dans l'éducation physique}

Au Brésil, le terme «genre» est apparu timidement à la fin des années 1980, pour ensuite devenir beaucoup plus fréquent (LOURO, 1996). Peu à peu, les études sur le genre se sont développées dans le domaine des sciences humaines. La traduction en langue portugaise en 1990 du texte GENDER. A Useful Category of Historical Analysis ${ }^{2}$, publié pour la première fois en 1986, constitue une référence importante pour les études sur le genre au Brésil, et il est largement utilisé dans le champ des sciences humaines et sociales. L'auteur y envisage le concept de genre comme une catégorie analytique et relationnelle liée à d'autres catégories telles que la race, la classe, la génération et la sexualité, entre autres. Il est aussi pensé comme une manière de donner du sens aux relations de pouvoir. La diffusion et la croissance des études sur le genre ont traversé les frontières disciplinaires ; en effet, le genre n'était plus seulement une catégorie d'analyse, il devenait un champ de connaissance important et reconnu dans les sciences humaines et sociales (MATOS, 2008).

En éducation physique, les recherches ayant adopté la catégorie Genre dans leurs analyses sont apparues au milieu des années 1990. Il y a bien eu avant cela des recherches se rapprochant de ce que l'on entend aujourd'hui par genre - études sur la femme, sur les classes mixtes d'éducation physique ou sur la co-éducation (ABREU, 1990) -, cependant le genre n'y était pas utilisé en tant que catégorie d'analyse. Il est une recherche sur le genre et l'éducation physique qui inaugure en quelque sorte les études en la matière : la thèse de doctorat d'Eustáquia SALVADORA DE SOUSA (1994), intitulée Meninos, à marcha! Meninas, à sombra! A Historia da Educação Física em Belo Horizonte (1897-1994) [Garçons, en marche ! Fillettes, à l'ombre ! L'histoire de l'éducation physique à Belo Horizonte (1897-1994)]. Il s'agit d'une recherche historique qui se sert du genre comme catégorie d'analyse pour réfléchir à l'histoire de l'éducation physique dans le milieu scolaire de la ville de Belo Horizonte, marquée par la ségrégation de filles et de garçons, d'hommes et de femmes. Le fait que cette recherche ait été menée dans une faculté d'éducation illustre combien le développement des études sur le genre et l'éducation physique est lié au domaine de l'éducation. Les études alliant jeunesse, genre et sexualité dans le domaine de l'éducation sont également très nombreuses au Brésil, comme le démontre une étude universitaire récente sur l'art et la jeunesse (SPOSITO et alii, 2009).

Cette production intense a été influencée par la fin de la séparation garçons/filles en cours d'éducation physique. Au fur et à mesure que les classes devenaient mixtes à partir des années 1990, un débat s'est instauré entre les enseignants, les personnes chargées des décisions scolaires et les chercheurs. Ont alors été analysés les avantages et désavantages, les conflits et les enjeux du nouveau système. L'étude de LOUZADA et alii (2007) souligne que les recherches sur les classes mixtes d'éducation physique réalisées entre 1990 et 2005 ont été menées dans un contexte universitaire très favorable à la mixité. À l'exception d'un seul travail qui défend la possibilité de variation dans les formes d'organisation des élèves selon le sexe, tous les autres soutiennent de manière explicite les classes mixtes. Il convient cependant d'ajouter que cette prise de position en faveur de 
la mixité dans les classes d'éducation physique ne coïncide pas nécessairement avec les dilemmes observés dans les écoles. Dans la réalité scolaire, les difficultés d'interaction entre les filles et les garçons restent encore importantes, et il n'est pas rare que certains enseignants finissent par séparer les garçons des filles au moment des exercices pratiques.

7 Ainsi, les garçons et les filles ont été séparés en deux groupes dans les cours d'éducation physique pendant très longtemps - et dans certains établissements, c'est encore le cas aujourd'hui. Cette séparation a été justifiée par le caractère pratique de la discipline, le fait de travailler avec le corps perçu sous une perspective biologique, ainsi que par l'organisation féminine et masculine de la plupart des compétitions sportives. D'un point de vue historique, cette ségrégation précède néanmoins l'hégémonie du sport comme contenu de la discipline de l'éducation physique. D'autre part, les études sur le genre ont mis l'accent sur le caractère naturel et biologique des corps et des différences entre hommes et femmes. Il serait faux de penser que le corps est seulement régi par des lois physiologiques qui échappent à l'histoire. Le corps et les relations de genre sont socialement produits, y compris dans les programmes scolaires.

Pour la biologiste Anne FAUSTO-STERLING (2005), il est nécessaire d'accepter le corps comme étant simultanément composé de gènes, d'hormones, de cellules et d'organes mais aussi de culture et d'histoire, qui ont une influence sur la santé et les comportements. L'auteur soulève des questions utiles pour les analyses développées ici : comment notre corps absorbe-t-il physiquement la culture ? De quelle manière notre expérience façonne-t-elle notre corps ? Comment parler du corps sans céder aux arguments de ceux qui le voient comme un objet naturel qui existe à part de la politique, de la culture et des changements sociaux?

9 Dans les années 1990, les cours d'éducation physique ont commencé à suivre l'organisation de classes déjà adoptée par les autres disciplines scolaires, devenant ainsi mixtes dans divers réseaux d'enseignement. Concrètement, un seul enseignant devenait responsable de toute la classe, garçons et filles réunis, pour la réalisation du cours. À partir de là, faire le choix de l'interaction entre les élèves ou les séparer à nouveau est devenu du ressort de l'enseignant ou de l'école.

Ces modifications en faveur de la mixité ont cependant rencontré de fortes résistances. À titre d'exemple, l'expérience des écoles municipales de la ville de Belo Horizonte ${ }^{3}$. Malgré l'arrêté du Secrétariat Municipal à l'Éducation qui stipulait la fin de la séparation filles/ garçons en classe en 1991, certains enseignants se sont mobilisés pour tenter de l'abroger (ALTMANN, 1998).

11 L'Association des Professionnels d'Éducation Physique de BELO HORIZONTE a adressé un recours au Conseil d'État d'Éducation qui, se basant sur la législation alors en vigueur, attestait de l'illégalité de la résolution. Elle affirmait que les cours d'éducation physique devaient «être adaptées à l'âge mais aussi au sexe des élèves, d'où la nécessité de séparer les filles des garçons pour la réalisation des exercices et des jeux» (SOUSA, 1994, p. 221). Malgré cet avis, le choix de la mixité a été maintenu.

Pour protester contre l'échec de l'abrogation, un grand nombre d'actions a été mené : arrêt total des activités, organisation des horaires scolaires dans le but de garantir des classes composées d'un nombre plus grand d'élèves du même sexe, répartition du temps du cours entre garçons et filles et même refus d'observer l'arrêté (SOUSA, 1994). 

garçons dans les écoles brésiliennes, et en particulier dans les cours d'éducation physique. Un autre facteur important dans cette résistance était lié au fait que les programmes de formation en éducation physique étaient différents pour les hommes et pour les femmes. Jusqu'en 1994, les disciplines pratiques du cours d'éducation physique de l'Université Fédérale de l'état de Minas GERAIS (UFMG) - seule université à Belo Horizonte à offrir une formation de professeurs d'éducation physique à l'époque n'étaient pas les mêmes suivant le sexe. Les enseignants n'ont pas été préparés pour faire face à une nouvelle organisation scolaire, et aujourd'hui encore l'approche de ces questions par la formation professorale reste questionnable. Au Brésil, travailler sur le genre dans les cours de formation d'enseignants brésiliens dépend fortement de la sensibilité de l'enseignant, car officiellement les programmes de formation mettant l'accent sur ce contenu sont peu nombreux ${ }^{4}$.

14 L'imposition de la mixité dans les classes a eu lieu en 1991, soit trois ans avant l'adoption d'un programme de formation enseignante unique par la Faculté d'Éducation Physique de l'UFMG. Jusqu'en 1994, les arguments basés sur les différences biologiques entre les sexes et sur les normes sportives nationales et internationales justifiaient les programmes de formation existants et, par conséquent, les séparations des filles et des garçons dans les cours d'éducation physique. Il serait pourtant erroné de croire que c'est le sport moderne qui a provoqué cette séparation; elle existait dans les programmes de formation bien avant qu'il soit adopté comme contenu d'éducation physique. En fait, les normes du sport moderne n'ont fait que légitimer et renforcer des valeurs déjà présentes :

Pour justifier le sexisme, l'éducation physique base en général son projet de séparation des sexes sur le corps perçu comme quelque chose de biologique, ainsi que sur la construction du corps féminin plus faible - par «nature» - que le masculin, renforçant le pouvoir des hommes sur les femmes sur l'échelle sociale. (SOUSA, 1994, p. 221)

Sans prétendre épuiser le sujet, nous allons à présent aborder certains défis que doit relever le milieu scolaire pour faire face à la mixité, en partant de deux axes d'analyse : l'occupation de l'espace physique scolaire et l'exclusion dans les sports.

\section{Occupation de l'espace physique scolaire}

Dans beaucoup d'établissements, les espaces scolaires ne sont pas occupés de la même façon par les filles et les garçons (THORNE, 1993, ALTMANN, 1998, Wenetz, 2006). Une recherche ethnographique (ALTMANN, 1998) menée dans un établissement public de Belo Horizonte avec des élèves âgés de 11 à 15 ans fréquentant quatre classes de $5 e$ série ${ }^{5} \mathrm{de}$ l'enseignement fondamental a révélé que les garçons occupaient des espaces plus vastes que les filles, à travers les pratiques sportives en général et le football en particulier. Tandis que la plupart des filles attendaient le début des cours en discutant entre elles sur les escaliers ou les bancs de l'école, plusieurs garçons simulaient des bagarres, 
improvisaient des jeux, couraient d'un côté à l'autre, jouaient au football... Rester immobile semblait extrêmement difficile pour les garçons, y compris en position assise ; ils se traînaient par terre pour échanger entre eux des coups de pied et des tapes. Pendant les récréations, l'occupation des terrains sportifs était différente selon le genre : les filles et les garçons jouaient ensemble à la balle au chasseur, par contre le football n'était pratiqué que par les garçons sur les deux terrains polysportifs de l'école.

Si de manière générale les garçons occupaient plus d'espace que les filles par l'intermédiaire du sport, ces divisions de l'espace selon le genre n'en étaient pas pour autant rigides. Parfois leurs frontières étaient franchies, comme l'a montré la présence de filles sur les terrains de foot:

Ce jour-là, elles sont arrivées en avance sur le terrain, avant même que retentisse la sonnerie, apportant avec elles un ballon. Lorsque les garçons sont arrivés, elles avaient déjà pratiquement constitué les équipes, prêtes à commencer le jeu et intransigeantes vis-à-vis de toute négociation. Deux garçons faisaient office d'arbitre, tandis que plusieurs autres attendaient autour la fin du match ou une occasion quelconque pour envahir le terrain. Voyant que la partie n'était pas sur le point de s'achever, trois d'entre eux se sont écriés:

"Si on peut pas jouer dehors, on va aussi jouer avec vous !». Elles ont alors cessé le match pour discuter et finalement argumenter qu'ils n'avaient qu'à jouer sur le terrain du haut, avec les autres garçons. Des gradins, on entendait crier répétitivement : «Garçon manqué!» (ALTMANN, 1998, p. 44)

21 Pour s'insérer dans cet univers masculin, les filles ont recouru à des stratégies. Tout d'abord, elles sont arrivées en avance sur le terrain avec un ballon pour éviter les conflits et se sont organisées avant l'arrivée des garçons. Ensuite, elles ont permis à deux d'entre eux d'être arbitres. En leur concédant ce rôle d'autorité, elles ont fait mine d'accepter la domination masculine de ce sport pour pouvoir jouer. Mais elles sont restées maitres des lieux, en accordant aux garçons le rôle d'arbitre et non pas l'exercice plein de cette fonction. Malgré les insultes qui fusaient, les arbitres - et non les joueuses - ont failli recevoir un carton rouge et être expulsés du terrain.

La résistance des filles à la domination masculine observée sur les terrains s'avéra si efficace que la situation s'inversa : elles dominaient et ils résistaient. S'ensuivit alors un effet de contre - résistance : en constatant qu'elles dominaient le terrain, les garçons tentèrent de récupérer leur statut en planifiant une invasion et en traitant les filles qui jouaient de garçons manqués ${ }^{6}$. Néanmoins, les «garçons manqués» ont joué pendant toute la récréation.

23 Même si les femmes pratiquent de plus en plus le football au Brésil depuis quelques années, le rapport entre football et masculinité persiste et n'efface pas l'idée reçue du lien entre joueuses et homosexualité - une tendance qui n'est cependant pas exclusivement brésilienne (CLARK \& PAECHTER, 2005). Pour Arlei DAMO (2005), le sport fonctionne comme une machine classificatoire qui hiérarchise les filles et les garçons. Le football s'étant naturalisé parmi les Brésiliens comme une pratique masculine, des attitudes 
différentes sont attendues des filles et des garçons. Dans la recherche ethnographique développée par l'auteur, on s'aperçoit que dire d'un garçon qu'il «est nul au foot» équivaut à affirmer qu'il n'est pas un vrai garçon. D'autre part, les filles tendent généralement à être exclues des matchs par les garçons, car aux dires de ces derniers leur présence empêche un vrai match ; si elles sont sur le terrain, ils ne peuvent exercer pleinement les hostilités qui leur sont propres.

\section{Exclusion dans les sports}

Les exclusions dans les jeux sportifs sont l'un des principaux motifs de conflits entre filles et garçons durant les cours d'éducation physique, et elles touchent les deux sexes (ALTMANN, 1998, 2005 ; CLARK \& PAECHTER, 2007 ; DORNELLES, 2009). Elles sont associées aux différences d'habiletés et d'implication par rapport aux sports. Si les analyses indiquent généralement une exclusion féminine des espaces de jeux sportifs, la recherche supra citée indique cependant qu'elles ne sont pas les seules à être exclues. Dans ce cas précis, la raison de leur exclusion n'était pas seulement liée au fait d'appartenir au sexe féminin, elles étaient en plus considérées comme étant plus faibles et moins habiles. L'âge, la force et l'habileté constituaient aussi des critères d'exclusion de certains garçons, comme l'illustre le témoignage de l'un des élèves : «J'suis nul au foot. J'suis pas bon. Quand le garçon m'a passé le ballon je l'ai pris et alors les garçons l'ont vu et ils m'ont pris le ballon. Ils m'ont demandé de sortir du terrain» (ALTMANN, 1998, p. 53). Le genre, la force, l'âge et l'habileté étaient donc les critères adoptés pour décider qui pouvait jouer ; des conditions surtout présentes au moment de la récréation, quand aucun enseignant n'intervenait pour garantir une plus grande égalité d'opportunités dans les pratiques sportives.

D'autre part, il existait une simultanéité entre être exclu et s'exclure, vu qu'il n'était pas toujours possible de savoir si le sujet était dans un coin du terrain parce qu'il ne recevait pas le ballon ou s'il ne recevait pas le ballon parce qu'il était placé dans un coin du terrain. Dans la crainte de commettre des fautes et de subir les commentaires des camarades, certaines filles et certains garçons se plaçaient un peu en retrait sur le terrain, comme s'ils préféraient ne pas recevoir le ballon plutôt que de courir le risque de ne pas réussir une passe. Cette situation s'observait aussi bien au football qu'au volleyball, les deux modalités sportives pratiquées dans cette école. Cela soulève la question de l'intervention de l'enseignant, comme le souligne l'enseignante de l'école observée :

Quel que soit le sport [...], on entend toujours : «untel a toujours le ballon». Alors apparaît une question, [c'est une] arme à double tranchant. Par exemple [...], cette fille qui ne touche jamais le ballon, [...] si je ne l'encourage pas à courir et à lutter pour son espace, elle ne va jamais [jouer]. Si je cède toujours et que je lui mets le ballon dans les mains, [...], elle ne va jamais se battre pour son espace. Elle doit aussi se battre pour l'espace, du moins verbaliser ce qu'elle ressent. Et encourager cette élève à courir après le ballon : "Si tu ne cours pas, tu ne vas jamais apprendre. On apprend seulement en faisant les choses, y a pas d'autres moyens ». En lui donnant quelques infos, pour que la personne puisse aussi s'aider ». (ALTMANN, 1998, p. 61) 
Sans se restreindre à la technique et à la tactique, l'éducation physique a besoin de favoriser le développement de ces habiletés qui sont indispensables pour la pratique d'un sport. Un tel processus éducatif doit prendre en compte les questions de genre existantes dans les pratiques sportives. D'après Robyne GARRET (2004), l'un des principaux défis de l'éducation physique est de trouver les moyens de donner aux filles et aux jeunes femmes la «capacitation» (empowerment) suffisante pour se sentir plus confiantes et habiles par rapport à l'utilisation de leur corps ; en somme, de leur éviter d'être confrontées aux formes restrictives de standards corporels de genre.

L'utilisation du sport comme base du programme de formation en éducation physique dans lequel les principes d'une masculinité hégémonique établissent des normes de réussite - situe les femmes comme moins capables, moins fortes, moins compétitives et moins habiles. À l'école, le fait que les élèves eux-mêmes souhaitaient que les pratiques et les espaces sportifs soient dominés par les garçons les plaçaient, d'une certaine manière, dans l'obligation d'être supérieurs aux filles. Mais cela n'était pas sans provoquer certaines gênes :

Pendant un cours, les filles jouaient au volley et à la balle au chasseur et les garçons au foot. Aline s'est dirigée vers le terrain de foot, mais les garçons ne voulaient pas la laisser jouer, sous prétexte qu'il y avait déjà beaucoup de monde. Voyant qu'elle ne renonçait pas, ROBSON lui a dit «alors, tu seras le goal». Mais Aline a refusé l'imposition, elle est restée à proximité jusqu'à ce que l'un d'eux consente à sa participation. Pendant le match, quand ROBSON a pris le ballon à Marcelo il a aussitôt crié : "Fais une passe à la fille, Marcelo! Fais une passe à la fille !». Peu de temps après, Aline a marqué un but et, à la fin du match, un autre garçon a dit à un de ses camarades : "Y a que toi qu'as pas marqué de but! Même Aline elle en a marqué un !» (ALTMANN , 1998, p. 69)

compte-rendu va dans le même sens que l'étude de DAMO (2005), à savoir que jouer avec les filles n'était pas pour les garçons un défi vu que faire une meilleure performance qu'elles ne leur apportait aucun mérite particulier. Par contre jouer moins bien était vécu comme une vexation dans la mesure où cela allait à l'encontre de la supériorité masculine attendue dans cet univers. Dès lors, jouer avec des filles ne représentait pas pour eux un défi, mais une menace.

Du côté des filles, dépasser les attentes et mieux jouer que les garçons était vécu comme un honneur, voire un motif de consécration qui leur garantissait parfois auprès de certains garçons une légitimité. À d'autres moments, la dévalorisation de leur pratique sportive et d'elles en tant que femmes était une manière de résister au trouble infligé par leur présence sur les terrains à la domination masculine de cet espace, comme quand elles étaient traitées de garçons manqués.

La séparation garçons/filles en cours d'éducation physique ou dans d'autres pratiques sportives masque un autre problème d'exclusion. Une construction du genre féminin 
n'est pas toujours possible dans un corps de sexe féminin, de même qu'une construction du genre masculin ne l'est pas toujours dans un corps de sexe masculin. Il est des constructions du féminin et du masculin qui suivent d'autres normes. Comment, au sein de l'école, faire face à un élève de sexe masculin qui se reconnaît comme fille? Ou de sexe féminin qui se reconnaît comme garçon ? Indépendamment de la question anatomique de l'organe sexuel, le corps peut être construit comme féminin ou masculin. À titre d'illustration, nous reproduisons ci-dessous le rapport d'un directeur d'une école de l'état de São Paulo sur une expérience qui a eu lieu en 2004 : joueurs. De plus, à la fin du rapport l'affirmation «seulement il est un garçon» indique que le sexe est considéré comme le définisseur de son genre. Même si Antonio s'habille, marche, se présente comme une fille et utilise un prénom féminin, son organe sexuel fait de lui un garçon. Dans ce raisonnement, le biologique est placé à l'origine et détermine le genre. Néanmoins, nous pouvons nous demander ce qui définit le plus son identité de genre : l'organe sexuel masculin ou ses désirs, ses sentiments, sa façon de s'habiller, de se comporter, etc. ? S'habiller, se comporter et se sentir fille ne seraient-ils pas plus déterminants pour son identité que ses organes sexuels ? Et comme il s'agit d'un championnat scolaire, l'organe sexuel masculin est-il aussi important pour décider à quelle équipe Maria devrait appartenir ? Le choix de Maria ne pourrait-il pas être le critère de définition de son équipe ? Ici la réponse est non, étant donné que la norme 
adoptée est une norme de genre hétérosexuelle, dans laquelle l'organe sexuel est utilisé comme critère.

D'un point de vue historique pourtant, les choses n'ont pas toujours été ainsi : les organes génitaux et sexuels n'ont pas toujours été considérés comme fondateurs du genre. L'historien Thomas LAQUEUR (2001) montre que la notion de différence sexuelle et l'idée de deux sexes biologiques distincts sont apparues entre le XVIIIe et le XIXe siècle. À partir de la fin du XVIIIe siècle, les significations de la différence sexuelle sont passées d'un modèle de sexe unique - où la femme était une version moins parfaite que l'homme - à un modèle fondé sur deux sexes, où la femme devenait le contraire de l'homme.

41 Jusqu'à la fin de la Renaissance, ledit sexe biologique ne constituait en fait pas un fondement solide du féminin et du masculin. Il n'y avait pas de sexe véritable et essentiel qui différenciait les hommes des femmes - et nous nous retrouvons là en face d'une question moderne. Pour LAQUEUR (2001), le sexe que nous connaissons (autrement dit les deux sexes en tant que nouveau fondement de genre) est apparu au XVIIIe siècle. Depuis, le corps est devenu un élément décisif :

Lorsque le corps a été perçu comme le représentant de la nature, il a assumé le rôle de "voix» de la nature ; autrement dit, quand a surgi le besoin de distinguer le féminin du masculin en des termes fortement binaires, le corps se devait de "parler» cette distinction de façon binaire. La conséquence fut une notion «bisexuée» du corps. (Nicholson, 2000, p. 21)

43 Ainsi, le genre et la sexualité ne peuvent être expliqués par le biais de déterminismes biologiques. La biologie n'explique pas à elle seule comment nous nous constituons comme hommes et femmes, ni de quelle manière nous vivons la sexualité. Ces expériences sont construites culturellement et historiquement.

\section{Considérations finales}

44 Les défis liés au genre sont très nombreux au sein d'une école qui propose de travailler conjointement avec les filles et les garçons, en particulier dans les cours d'éducation physique. De tels défis concernent les pratiques de signification et les rapports de pouvoir notamment présents dans les programmes scolaires et les programmes de formation professionnelle. Réunir filles et garçons dans un même cours d'éducation physique ne met fin à aucun conflit, dans certains cas cela va même jusqu'à les rendre plus évidents. Ce nonobstant, l'organisation de classes mixtes montre que dans un cours d'éducation physique d'autres éléments que les seules différences sexuelles sont en jeu. Les classes mixtes brisent une division extrême et unique entre le féminin et le masculin, tout en considérant les variations dans les genres, en diluant les frontières et en permettant de les franchir. Restent aux écoles, aux enseignants et aux élèves la tâche de relever ces défis. 


\section{BIBLIOGRAPHIE}

ALTMANN, Helena. (1998). Rompendo fronteiras de gênero: Marias (e) homens na Educação Física. Mémoire de Master en Éducation, Université Fédérale de l'état de Minas Gerais (UFMG).

ALTMANN , Helena. (2005). Pedagogias da sexualidade e do gênero: educação sexual em uma escola. Thèse de doctorat en éducation. Université Pontificale Catholique (PUC) de Rio de Janeiro. Disponible sur le site Internet http://www2.dbd.pucrio.br/pergamum/biblioteca/php/ mostrateses.php?open=1\&arqtese=0114341_05_Indice.html, consulté le 25/05/2009.

CLARK, Sheryl et PAECHTER, Carrie. (2007). «Why can't girls play football?» Gender dynamics and the playground. Sport, Education and Society, vol. 12, no 3, pp. 261-276.

DAMO, Arlei Sander. (2005) Do dom à profissão: o futebol de espetáculo em perspectiva etnográfica a partir da formação/produção de atletas profissionais no sul do Brasil. Thèse de doctorat en anthropologie sociale, Université Fédérale du Rio Grande do Sul (UFRGS), Porto Alegre. Disponible sur le site Internet http://www.bibliotecadigital.ufrgs.br/da.php? nrb=000468905\&loc=2005\&l=24a7bc666aac4e57, consulté le 28/01/2010.

DORNELLES, Priscila G. (2009) A separação de meninos e meninas na educação física escolar: marcas de gênero. Anais do XVI CONBRACE. Disponible sur le site Internet : http://www.rbceonline.org.br/ congressos/index.php/CONBRACE/XVI/ paper/view/349/539, consulté le 28/01/2010.

FAUSTO-STERLING, Anne. (2005) The Bare Bones of Sex: Part 1 - Sex and Gender. Journal of Women in Culture and Society. vol. 30, nº 2, pp. 1491-1527.

GARRETT, Robyne. (2004) Negotiating a Physical Identity: girls, bodies and physical education. Sport, Education and Society, vol. 9, no 2, pp. 223-237.

Gênero e diversidade na escola. (2009). Formação de professoras/es em gênero, sexualidade, orientação sexual e relações étnico-raciais. Livro de Conteúdos. Versão 2009. Rio de Janeiro : CEPESC ; Brasília : SPM. Disponible sur le site Internet http://sistema.clam.org.br/biblioteca /? q=node/500, consulté le 28/01/2010.

LAQUEUR, Thomas. (2001). Inventando do sexo. Corpo e gênero dos gregos a Freud.Traduction de Vera Whately. Rio de Janeiro : Relume-Dumará.

LOURO, Guacira Lopes. (1997). Gênero, sexualidade e educação - uma perspectiva pós-estruturalista. Petrópolis : Vozes.

LOUZADA, Mauro, VOTRE, Sebastião et DEVIDE, Fabiano. (2007). Representações de docentes acerca da distribuição dos alunos por sexo nas aulas de educação física. Revista Brasileira de Ciências do Esporte. Campinas, vol. 28, no 2, janvier, pp. 55-68.

NICHOLSON, Linda. (2000) Interpretando o gênero. Revista de Estudos Feministas, an 8, no 2, pp. 9-41, 20 semestre.

PEREIRA, Maria Elisabete et alii. (2007) Gênero e diversidade na escola. Formação de professoras/es em gênero, sexualidade, orientação sexual e relações étnico-raciais. Brasília/Rio de Janeiro : SPM/CEPESC. Disponible sur le site Internet http://sistema.clam.org.br/biblioteca/ ?q=node/405, consulté le 28/01/2010. 
SOUSA, Eustáquia Salvadora DE. (1994) Meninos, à marcha! Meninas, à sombra! A história da Educação Física em Belo Horizonte (1897-1994). Thèse de doctorat en éducation, Université de Campinas (UNICAMP). Disponible sur le site Internet http://libdigi.unicamp.br/document/? code=vtls000083959, consulté le 28/01/2010.

WENETZ, Ileana, STIGGER, Marco P. et MEYER, D. E. (2006) A construção do Gênero no espaço Escolar. Movimento : Porto Alegre, vol. 12, pp. 59-80.

\section{NOTES}

1. L'expression " classes mixtes " se réfère uniquement à l'organisation de classes composées de filles et de garçons, à la différence des classes divisées selon le sexe. Dans ce cas, c'est l'enseignant qui décide si les garçons et les filles peuvent pratiquer des activités ensemble ou séparément. Le terme " co-éducation » concerne les classes d'éducation physique suivies par des filles et des garçons ensemble, qui interagissent entre eux au cours des pratiques sportives.

2. Traduit en français en 1988, sous le titre Le genre : une catégorie utile de l'analyse historique.

3. Capitale de l'état de Minas Gerais, Belo Horizonte est la cinquième ville du Brésil, avec 2452 617 habitants. Site Internet http://www.gov.br/cidadesat/topwindow.html., consulté le 26/01/2010.

4. D'un autre côté, les cours de formation continue sur cette thématique sont relativement fréquents, ce qui montre que dans le quotidien scolaire les enseignants sont confrontés aux problématiques du genre et de la sexualité. À titre d'exemple, le cours de formation à distance Genre et Diversité à l'École : formation d'enseignants sur le genre, la sexualité, l'orientation sexuelle et les relations ethniques et raciales. Un cours issu du partenariat entre le gouvernement brésilien, le British Council et le Centre Latino-Américain sur la Sexualité et les Droits de l'Homme (important centre de recherche sur le sujet) (Pereira, 2007). Voir aussi Genro e Diversidade na escola, Pereira, 2009.

5. La 5e série de l'enseignement fondamental correspond approximativement au CM2 en France.

6. Dans la langue portugaise, l'épithète utilisé est Maria-Homem, littéralement « Marie-Homme ».

\section{RÉSUMÉS}

Les années 1990 ont profondément modifié la recherche et les pratiques scolaires liées au genre, à l'éducation et à l'éducation physique. Au Brésil, la mise en place de la mixité dans les cours d'éducation physique donna lieu à l'apparition d'études sur le genre. Le présent article étudie certains des défis que doit relever le milieu scolaire en face de cette configuration mixte de l'éducation physique, et ce à partir de deux axes d'analyse : l'occupation de l'espace physique scolaire et les exclusions dans les sports.

The nineties brought about significant changes in the fields of research and school practices on gender, education and physical education. In Brazil, physical education classes became mixed and gender studies emerge. This article analyzes some of the school challenges from this mixed configuration of physical education classes, and this is done through two foci of analysis: the occupation of the school's physical space and exclusions in sports. 
INDEX

Keywords : gender, physical education, school

Mots-clés : école, éducation physique, genre, sport

\section{AUTEUR}

\section{HELENA ALTMANN}

Professeur de la Faculté d'éducation physique de l'Université de Campinas (UNICAMP), Brésil 